\title{
Blame the Disorder
}

Can J Neurol Sci. 2014; 41: 141

The portrayals of Tourette syndrome (TS) in film and TV are typically exaggerated and often unhelpful. Clinicians may need to counteract the resulting distorted image for their patients and their families as discussed in this issue of the Journal. ${ }^{1}$ Whether the purveyors of entertainment can be expected to respond to calls for more realistic presentations is doubtful. The problem is arguably compounded by confusion that is inherent in the nature of the disorder as we presently know and represent it, and trying to convert an arbitrary operational research-oriented definition into one comprehensible by the public. This problem may be fundamentally insoluble, though some aspects are subject to improvement.

Each of the following components of the confusion can have actual consequences, and this is not an exhaustive exposition. In a forthcoming book ${ }^{2}$ this argument will be further elaborated.

1. What are tics? Not all are brief (some are dystonic and prolonged) and some may be rhythmic. There is no necessary daily number, and many go unnoticed. They are not purely motor disorders, but have an important and sometimes predominant sensory component.

2. Definition of tic disorders. In DSM-IV, DSM-IV-TR, and now DSM5, there is an unexplained arbitrary criterion for TS requiring at least two motor tics and at least one vocal tic, persisting for a year or more. As soon as number and kind are invoked, boundary issues arise. The history of the specific $2+1$ is lost and its persistence unjustified. The boundary of 'vocal' is unclear and regularly violated by equating it with 'phonic' or even 'verbal.' Worse is that a common repetitive behaviour can be assumed to be a tic (e.g., joint-cracking, bruxism, finger-smelling, thumb-sucking) to fulfill the minimum $2+1$ criteria.

3. Genetics. Tics run in families, but the TS entity defined by a committee cannot itself be inherited. The experience is real, but not the definition. "Things perceived as real are real in their consequences." Sometimes services a patient receives may depend upon having the specific TS label, not that of another tic category.

4. Comorbidity. About $85 \%$ of persons with what we call TS are comorbid with (on average) two other neuro-developmental disorders, most commonly ADHD and OCD, ${ }^{3}$ whose behavioural symptom boundaries overlap tics and vary over time. Impairment or severity due specifically to tics is often impossible to judge, and treatment of one of the three may alter the manifestations and severity of one or more of the others.

There can be actual consequences, such as:

1. Because most publications presented data on TS, those who didn't quite qualify, who would now be said to fulfill criteria for persistent (motor or vocal) tic disorder have at times erred in assuming that there is no treatment for them because they don't 'have' TS.
2. Some persons in whose family there was someone with very significant TS conclude that if they have a child there is a high risk of having an equally affected child with TS (not just a tendency for a tic disorder) and may elect to have no children.

3. The severity assumption ("TS is the most severe of the tic disorders") often means that clinicians prefer to be nice and tell parents within the first 12 months or for some time thereafter that their child has tics that are "benign" or "simple" and not TS. Sooner or later other kinds of tics occur or one of them evolves into a complex tic and then the dreaded TS diagnosis is applied. This not at all uncommon experience reinforces to the parents and their friends and families that now their child has a seemingly progressive neurological condition that is necessarily "serious". But this may be wrong. Although by definitional criteria on average TS may be more complex than other tic disorders, its severity or degree of impairment will largely depend upon individual social and psychological factors. The negative social impact of a single tic can be worse than that of mild TS. Furthermore, becoming worse before better is a common developmental trajectory for childhood TS.

In conclusion, then, as a disorder with many difficulties in establishing and maintaining diagnostic criteria that will exist in a public as well as research space, have a fuzzy boundary with other disorders, highly variable symptoms in childhood with important and often crucial interactions with one's social environment, the essence of what we mean by Tourette syndrome (at least for the public) cannot be adequately captured and therefore our expectations of public education should be limited. At the same time, credit is due the Tourette's category for providing a challenging insight into how humans can function and be helped to function better when all fields of knowledge are brought to bear, when uncertainty is acknowledged, and when humility is sustained.

\section{Roger D. Freeman, Neuropsychiatry Clinic, BC Children's Hospital and University of British Columbia, Vancouver, BC, Canada.Email: rfreeman@cw.bc.ca}

\section{REFERENCES}

1. Calder-Sprackman S, Sutherland S, Doja A. The portrayal of Tourette syndrome in film and television. Can J Neurol Sci. 2014;41(2):226-32.

2. Freeman, RD. Tics and Tourette syndrome: key clinical perspectives. Clinics in developmental medicine. London, UK: Mac Keith Press; Forthcoming 2014.

3. Freeman RD, Fast DK, Burd L, Kerbeshian J, Robertson M, Sandor P, Tourette Syndrome International Database Consortium. An international perspective on Tourette syndrome: selected findings from 3,500 individuals in 22 countries. Dev Med Child Neurol. 2000;42(7):436-47. 